\title{
A IMPORTÂNCIA DA ESTATÍSTICA DESCRITIVA NA PANDEMIA DE COVID-19
}

\section{ARTIGO ORIGINAL}

LACERDA, Fábio Henrique De Souza ${ }^{1}$

LACERDA, Fábio Henrique De Souza. A importância da estatística descritiva na pandemia de Covid-19. Revista Científica Multidisciplinar Núcleo do Conhecimento. Ano 05, Ed. 08, Vol. 02, pp. 05-14. Agosto de 2020. ISSN: 2448-0959, Link de acesso: https://www.nucleodoconhecimento.com.br/matematica/estatistica-descritiva

\section{RESUMO}

Com o avanço da pandemia de covid-19 no mundo, vários países tiveram que adotar medidas restritivas rígidas para tentar parar a propagação desse vírus. Por isso, o objetivo desse trabalho é de mostrar a importância da estatística descritiva e de como têm auxiliado esses países a tomarem decisões. O desenvolvimento dessa pesquisa bibliográfica terá como base, autores nacionais e internacionais, sendo utilizadas pesquisas em livros e artigos no período entre 1980 e 2020. A estatística sempre foi fundamental em todas as áreas do conhecimento e hodiernamente estamos vendo o quanto ela tem contribuído no combate do covid-19.

Palavras-Chave: Estatística descritiva, covid-19, importância da estatística.

\section{INTRODUÇÃO}

Desde do novo surto de coronavírus (SARS-CoV-2), que teve origem na China, o causador da Covid-19, vem trazendo grande inquietação diante da população, que

${ }^{1}$ Graduando Em Licenciatura Plena Em Matemática E Graduando No Curso Superior Tecnólogo Em Gestão Financeira. 
paralisou grande nações e se espalhou aceleradamente em diversos lugares no mundo, com diferentes impactos (FREITAS; NAPIMOGA; DONALISIO, 2020)

E para ajudar a combater esse vírus, a matemática tem sido uma das principais áreas de auxílio. Pois, para Chaoubah (2020), os modelos matemáticos são importantes para conseguir estimar a quantidade de casos em vários cenários, assim, auxiliando para que muito dos tomadores de decisão consiga chegar a uma solução objetiva, por exemplo, podendo ajudar a determinar o número de leitos disponíveis em hospitais em determinadas regiões.

Sendo assim, uma das áreas especificas da matemática que ajuda no processo de combate ao Covid-19 é a estatística, que sucede em uma ferramenta imprescindível em todas as ciências, sejam elas exatas, saúde ou humanas.

Segundo Crespo (2002), a estatística é uma das áreas da matemática aplicada muito importante, tendo como objetivos específicos fornecer métodos para coleta, descrição, organização, sendo esses citados acima faz parte da estatística descritiva. E a análise e interpretação de dados, faz da parte da estatística inferencial.

Objetiva-se com esse trabalho, ressaltar a importância da estatística descritiva no enfrentamento do covid-19 e também, de como têm auxiliado esses países á tomarem decisões. Para isso, utilizaremos de pesquisa bibliográficas em livros e principalmente de artigos científicos.

\section{ESTATÍSTICA DESCRITIVA}

Segundo Costa (2011, p.19), define estatística descritiva como:

É aquela que possui um conjunto de técnicas para planejar, organizar, coletar, resumir, classificar, apurar, descrever, comunicar e analisar os dados em tabelas, gráficos ou em outros recursos visuais, além do cálculo de estimativas de parâmetros representativos desses dados, interpretação de coeficientes e exposição que permitam descrever o fenômeno 
A princípio, estatística descritiva tem como característica principal a organização de dados, mas as vezes acabamos nos deparando com uma grande massa de dados e a organização não se torna uma tarefa simples como podemos imaginar.

Essa área da estatística é conhecida por ser usada em situações em que são encontradas uma grande quantidade de informações, sendo necessária torná-las compactas para conseguir trabalhar com os dados. (PEREIRA, 2019).

\section{A IMPORTÂNCIA DA ESTATÍSTICA}

Segundo David Goggon (2015), os procedimentos estatísticos têm contribuído em muitos dos sucessos da medicina moderna, salvando muitas vidas. Por isso, que o máximo de profissionais de saúde deveriam conhecer, pelo menos, os mecanismos básicos relevantes.

E assim, a estatística tem ajudado os profissionais da saúde, para o enfrentamento de tomadas de decisões nessa pandemia, para Chauobah, as análises estatísticas apresentaram que algumas medidas usadas para diminuir o número de contagio do coronavírus tiveram resultado na redução de casos, que por exemplo, o mais adequado foram as políticas de afastamento social. Foram elas que fizeram os governos adotarem essa medida de restrição, que por sua vez, mostrou um melhor diagnóstico e alguns dos países que adotaram essa medida foram Itália e Reino Unido.

É com base nos dados estatísticos, que os governos têm formado discussões em busca de estratégias eficientes de combate à propagação do vírus.

\section{OS RECURSOS MAIS UTILIZADOS DA ESTATÍSTICA DESCRITIVA}

Do ponto de vista de Guedes e tal, (2005, p.1):

A estatística descritiva, cujo objetivo básico é o de sintetizar uma série de valores de mesma natureza, permitindo dessa forma que se tenha uma visão global da variação desses valores, organiza e descreve os 
dados de três maneiras: por meio de tabelas, de gráficos e de medidas descritivas.

\subsection{TABELAS}

A tabela é uma das formas mais fácil de mostrar e de resumir os dados, sendo assim, ficando melhor para observar e interpretar os valores. O intuito dela, é apresentar dados com clareza e de rápida compreensão (CALVO, 2004).

\subsubsection{TABELA PRIMITIVA}

As tabelas conhecidas como primitiva e rol, são utilizadas nas exibições de dados que não apresenta uma organização dos números, que também, é conhecida como dados brutos.

Tabela 4.1- A quantidade de óbitos por covid-19 no Brasil diariamente em 30 dias, desde do dia 17/04/20 até 16/05/20.

\begin{tabular}{|l|l|l|l|l|l|}
\hline Data & Quantidade & Data & Quantidade & Data & Quantidade \\
\hline $\mathbf{1 7 / 0 4}$ & 210 & $27 / 04$ & 310 & $07 / 05$ & 610 \\
\hline $\mathbf{1 8 / 0 4}$ & 216 & $28 / 04$ & 496 & $08 / 05$ & 751 \\
\hline $\mathbf{1 9 / 0 4}$ & 109 & $29 / 04$ & 434 & $09 / 05$ & 730 \\
\hline $\mathbf{2 0 / 0 4}$ & 116 & $30 / 04$ & 428 & $10 / 05$ & 496 \\
\hline $\mathbf{2 1 / 0 4}$ & 167 & $01 / 05$ & 442 & $11 / 05$ & 396 \\
\hline $\mathbf{2 2 / 0 4}$ & 166 & $02 / 05$ & 396 & $12 / 05$ & 881 \\
\hline $\mathbf{2 3 / 0 4}$ & 403 & $03 / 05$ & 294 & $13 / 05$ & 749 \\
\hline $\mathbf{2 4 / 0 4}$ & 368 & $04 / 05$ & 277 & $14 / 05$ & 844 \\
\hline $\mathbf{2 5 / 0 4}$ & 359 & $05 / 05$ & 600 & $15 / 05$ & 824 \\
\hline $\mathbf{2 6 / 0 4}$ & 197 & $06 / 05$ & 615 & $16 / 05$ & 816 \\
\hline
\end{tabular}

Fonte: Secretarias Estaduais de Saúde. Brasil, 2020 
Assim, nessa tabela primitiva podemos perceber que ela não tem nenhuma ordem, e fica difícil para quem está visualizando descobrir qual dia que ocorreu uma quantidade maior e menor de óbitos. Ela contém os dados bruto sem nenhuma organização.

\subsubsection{ROL}

Segundo Waldir Medri (2011), pelo rol, conseguimos de maneira mais clara e ágil a composição do conjunto, verificando com rapidez o maior e o menor valor dos números, além de alguns dados que pode se repetir muitas vezes. Dessa forma, podendo obter o comportamento dos dados com mais agilidade.

Tabela 4.2 - A quantidade de óbitos por covid-19 no Brasil diariamente em 30 dias, desde do dia 17/04/20 até 16/05/20.

\begin{tabular}{|l|l|l|l|l|l|}
\hline Data & Quantidade & Data & Quantidade & Data & Quantidade \\
\hline $\mathbf{1 9} / \mathbf{0 4}$ & 109 & $25 / 04$ & 359 & $05 / 05$ & 600 \\
\hline $\mathbf{2 0 / 0 4}$ & 116 & $24 / 04$ & 368 & $07 / 05$ & 610 \\
\hline $\mathbf{2 2} / \mathbf{0 4}$ & 166 & $02 / 05$ & 396 & $06 / 05$ & 615 \\
\hline $\mathbf{2 1 / 0 4}$ & 167 & $11 / 05$ & 396 & $09 / 05$ & 730 \\
\hline $\mathbf{2 6 / 0 4}$ & 197 & $23 / 04$ & 403 & $13 / 05$ & 749 \\
\hline $\mathbf{1 7 / 0 4}$ & 210 & $30 / 04$ & 428 & $08 / 05$ & 751 \\
\hline $\mathbf{1 8} / \mathbf{0 4}$ & 216 & $29 / 04$ & 434 & $16 / 05$ & 816 \\
\hline $\mathbf{0 4} / \mathbf{0 5}$ & 277 & $01 / 05$ & 442 & $15 / 05$ & 824 \\
\hline $\mathbf{0 3 / 0 5}$ & 294 & $28 / 04$ & 496 & $14 / 05$ & 844 \\
\hline $\mathbf{2 7 / 0 4}$ & 310 & $10 / 05$ & 496 & $12 / 05$ & 881 \\
\hline
\end{tabular}

Fonte: Secretarias Estaduais de Saúde. Brasil, 2020

Agora, com relativa facilidade podemos saber qual o valor mínimo e máximo de óbitos nesse período de tempo e determinar qual foi data de ocorrência.

\subsection{GRÁFICOS}

Segundo Crespo, (2002, p.21) define gráfico como: 
O gráfico estatístico é uma forma de apresentação dos dados estatístico, cujo objetivo é o de produzir, no investigador ou no público em geral, uma impressão mais rápida e viva do fenômeno em estudo, já que os gráficos falam mais rápido.

Os gráficos são uma das ferramentas mais utilizadas pela mídia para mostrar a dispersão dos valores. Facilita na compreensão do público, pois consegue a impressão mais rápida dos dados que está sendo expostos.

Existem diversos tipos de gráficos, como por exemplo, gráfico em linha, gráfico em barra, gráfico setorial, entre outros. Mas, o gráfico a ser apresentado aqui é o de setores.

\subsubsection{GRÁFICO SETORIAL}

Sônia Viera (1980, p.20), explica alguns detalhes desse gráfico;

Para fazer um gráfico de setores, primeiro se traça uma circunferência que, como se sabe, tem $360^{\circ}$. Essa circunferência representa o total, ou seja $100 \%$. Dentro dessa circunferência devem ser representadas as categorias da variável em estudo.

Exemplo: Quantidade de casos confirmados de covid-19 por regiões do Brasil, até o dia 15/07/20.

Tabela 4.3 - Quantidade de casos confirmados de covid-19 por regiões do Brasil, até o dia 15/07/20.

\begin{tabular}{|l|l|}
\hline Regiões & $\begin{array}{l}\text { Quantidade de } \\
\text { casos }\end{array}$ \\
\hline Centro-Oeste & 159.224 \\
\hline Sul & 137.339 \\
\hline Norte & 334.487 \\
\hline Nordeste & 659.711 \\
\hline Sudeste & 675.987 \\
\hline Total & 1.966 .748 \\
\hline
\end{tabular}


Fonte: Secretarias Estaduais de Saúde. Brasil, 2020

Desse jeito, temos que:

Figura 4.1

\section{Gráfico setorial: quantidade de casos confirmados de covid-19 por regiões no Brasil , até o dia 15/07/20}

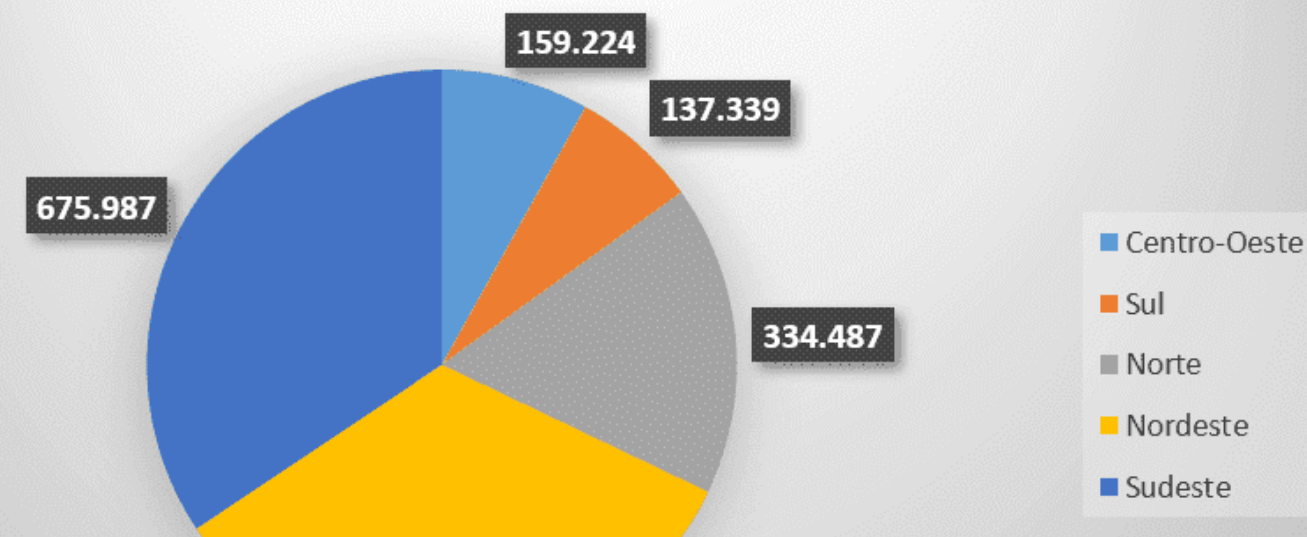

659.711

Fonte: Secretarias Estaduais de Saúde. Brasil, 2020

\subsection{MEDIDAS DE TENDÊNCIA CENTRAL}

Para Azevedo (2016, p.142), define essa medida como:

As medidas de tendência central ou pro médios são valores que servem para representar a distribuição como um todo, além de possibilitarem o confronto entre distribuições. Das principais medidas de tendência central destacamos aqui a média aritmética e a mediana.

\subsubsection{MÉDIA ARITMÉTICA}

A média aritmética simples ou conhecidamente apenas média, é obtida através de um conjunto de dados, basta dividir o total da soma de valores do conjunto pela quantidade de elementos que esse ele possui (FALCO E JUNIOR, 2012) 


\section{MULTIISCIPLINARY SCIENTIFIC JOURNAL REVISTA CIENTÍFICA MULTIDISCIPLINAR NÚCLEO DO

\section{$\bar{x}=\frac{\text { Soma de todos os valores }}{\text { Número de dados somados }}$}

\subsubsection{MEDIANA}

Maytê Pereira (2019) enfatiza que a mediana é a medida de posicionamento central, de maneira simples é o valor que estiver no meio de um conjunto de dados que estejam colocados em ordem crescentes ou decrescentes.

Se pôr a caso a quantidade de dados for ímpar, fica bem mais fácil de achar a mediana, pois ela será o número que estiver no centro do conjunto. Se a quantidade de dados for par, vai ser necessário encontrar a média dos dois valores que estiverem no centro e assim, o valor encontrado é a mediana.

\subsubsection{MODA}

A moda de um conjunto de dados é o valor que ocorre com a maior frequência. Um conjunto de dados pode ter uma moda, mais de uma moda, ou não ter moda (LARSON; FARBER, 2015).

\section{CONCLUSÃO}

A estatística vem fazendo a diferença no meio dessa pandemia, contribuindo de maneira expressiva. Tem auxiliado os profissionais da saúde e de outras áreas para o enfrentamento de tomadas de decisões

Com as ferramentas da estatística descritiva, vimos o quanto ela está presente no combate do covid-19. Foi mostrado através de exemplos que ela tem sido fundamental nas estratégias de planejamento, tomadas de decisões e de organização de dados.

Portanto, é necessário ter conhecimento da importância dessa área da estatística, pois ela tem sido mostrada na mídia diariamente, através de tabelas, gráficos e de 
medidas descritivas. Por isso, ela merece toda a atenção nesse momento difícil que o país vem sofrendo com a quantidade de mortes.

\section{REFERÊNCIAS}

AZEVEDO, Paulo Roberto Medeiros de. Introdução à estatística [recurso eletrônico] / Paulo Roberto Medeiros de Azevedo. - 3. ed. - Natal, RN : EDUFRN, 2016.

CALVO, M. C. M. Estatística descritiva. Florianópolis: UFSC, 2004.

COGGON, David - a importância da estatística na pesquisa em saúde, 2015.

COSTA, Paulo Roberto da. Estatística. Santa Maria: Universidade Federal de Santa Maria, Colégio Técnico Industrial de Santa Maria, Curso Técnico em Automação Industrial, 3. ed., 2011.

CRESPO, Antônio Arnot. Estatística fácil - 17. ed - São Paulo. Saraiva, 2002

DIEHL, C. A.; SOUZA, M. A.; DOMINGOS, L. E. C. O uso da estatística descritiva na pesquisa em custos: análise do xiv congresso brasileiro de custos, Porto Alegre, v. 7, n. 12, 2o semestre 2007.

FALCO, Javert; JUNIOR, Roberto. Estatística. Curitiba - PR, 2012

FREITAS, ARF; NAPIMOGA, MH; DONALISIO, MR. Análise da gravidade da pandemia de Covid-19; Universidade Estadual de Campinas, Departamento de Saúde Coletiva, Campinas, SP, Brasil.

LARSON, Ron. Estatística aplicada / Ron Larson, Betsy; Farber ; tradução José Fernando Pereira Gonçalves; revisão técnica Manoel Henrique Salgado. -- São Paulo : Pearson Education do Brasil, 2015.

MEDRI, Waldir. Análise exploratória de dados. Londrina/PR, 2011. 
Painel de casos de doença pelo coronavírus 2019 (COVID-19) no Brasil pelo Ministério da Saúde: disponível em <https://covid.saude.gov.br/>

PEREIRA, Maytê - Estatística Descritiva Básica: o que é e para que serve,2019.

UFJF NOTICÍAS. Pesquisador explica como a estatística ajuda no combate à Covid-19. 2020.2 Disponível em: <https://www2.ufjf.br/noticias/2020/04/22/pesquisador-explica-como-a-estatisticaajuda-no-combate-a-covid-19/>.

VIERA, Sonia. Introdução á Bioestatística - 3 ed. Revista e 3 ed. Ampliada, - Rio de Janeiro: Elsevier, 1980 - $16^{\circ}$ reimpressão.

Enviado: Julho, 2020.

Aprovado: Agosto, 2020. 